\title{
Fast lithium-beam spectroscopy of tokamak edge plasmas
}

\author{
E. Wolfrum, a) F. Aumayr, D. Wutte, and HP. Winter \\ Institut für Allgemeine Physik, Technische Universität Wien, Austria \\ E. Hintz, D. Rusbüldt, and R. P. Schorn \\ Institut für Plasmaphysik, KFA Jülich GmbH, Ass. Euratom-KFA, Germany
}

(Received 30 December 1992; accepted for publication 26 April 1993)

\begin{abstract}
Plasma-wall interaction and impurity transport processes in the outermost region of magnetically confined hot plasmas (the so-called plasma edge) must be well understood for successful development of future thermonuclear fusion reactors. To this goal, sufficiently detailed edge plasma diagnostics are in great demand. By injecting a fast $\mathrm{Li}$ beam into the edge plasma region, a great number of information can be obtained with excellent space and time resolution. This so-called Li-beam plasma spectroscopy gives access not only to edge plasma density profiles from the collisionally excited $\mathrm{Li}$ atoms, but also to the impurity concentration and temperature profiles via line emission induced by electron capture from the injected $\mathrm{Li}$ atoms by the impurity ions. Full utilization of all capabilities requires a reliable data base for the atomic collision processes involving injected $\mathrm{Li}$ atoms and plasma constituents (i.e., electrons, hydrogen ions, and relevant impurities in their various charge states), since a precise modeling of $\mathrm{Li}$ beam attenuation and excited-state composition has to be made for evaluating desired plasma properties from the related spectroscopical measurements. The most recent methodical improvement permits a fully consistent determination of absolute edge plasma density profiles by measuring only relative LiI line emission profiles. This is of special interest for investigating rapid edge plasma density fluctuations in conncction with, e.g., ELMs, $L-H$ mode transition, turbulence or edge cooling by impurity injection. This paper describes the capabilities of Li-beam edge plasma spectroscopy by way of illustrative examples from measurements at the tokamak experiment TEXTOR.
\end{abstract}

\section{LI-BEAM PLASMA SPECTROSCOPY_PRINCIPLES OF OPERATION}

The production of hot, dense magnetically confined plasmas for thermonuclear fusion studies relies to a great deal on the understanding of reaction kinetics and transport phenomena in the plasma boundary (the so-called edge region). Due to plasma-wall interaction the edge plasma will become contaminated, which gives rise to energy and particle losses as well as local and global plasma instabilities. All these processes influence again the plasmawall interaction, and so on. Due to these interrelations a quite detailed understanding of the plasma edge behavior is made difficult and can only be gleaned from accurate measurements of all relevant edge plasma parameters.

The so far common plasma diagnostic techniques, in particular "passive" photon emission spectroscopy throughout the electromagnetic spectrum, are not ideally suited for investigations in the plasma edge because of its relatively small extension and strong inhomogeneity. Conditions in the plasma edge are determined by a large number of interrelated atomic collision processes, which necessitates extensive plasma modeling for extraction of any information of interest from the diagnostic raw data. Such modeling processes give rise to considerable ambiguities. Better edge plasma diagnostics are thus needed and should comply with the following requirements: Excellent spatial

\footnotetext{
a) Also at Institut für Plasmaphysik, KFA Julich GmbH, Ass. EuratomKFA, Germany.
}

( $<\mathrm{cm})$ as well as temporal $(\leqslant \mathrm{ms})$ resolution, minimum introduction of plasma disturbance, evaluated plasma parameters should not strongly depend on the modeling procedures.

All these goals can be met by fast Li-beam plasma spectroscopy, i.e., the injection of $\mathrm{keV}$ to multi-keV neutral lithium beams into the plasma edge, to induce collisional processes which deliver the desired diagnostic informations. As an example, the plasma electron density can be detcrmined by observing the LiI resonance line emission along the injected beam. ${ }^{1-4}$ In close relation to this, magnetic field distributions inside a tokamak discharge can be determined ${ }^{5,6}$ by observing Zeeman splitting of the electron impact-induced LiI resonance line. By combining Li beam injection with laser-induced fluorescence and injection of other atomic species, the electron temperature can be measured. ${ }^{7,8}$ Furthermore, electric field strengths can be determined by observing optically forbidden LiI transitions as excited by dyc laser radiation., 90

All of the so far mentioned diagnostic applications rely on photon emission from electron impact-excited Li atoms [Li impact excitation spectroscopy ( $\mathrm{Li}-\mathrm{IXS}$ )]. We point to the fact, however, that also collisions with protons and impurity ions lead to LiI excitation, ${ }^{11}$ which is of relevance for quantitative evaluation of Li-IXS data (see below).

A second class of atomic collisions relevant for fast Li-beam plasma spectroscopy involves electron capture from the injected $\mathrm{Li}$ atoms by plasma protons and/or impurity ions, which produces excited states of the latter. In a conceptually equivalent way, fast $\mathrm{H}$ atom beams are al- 


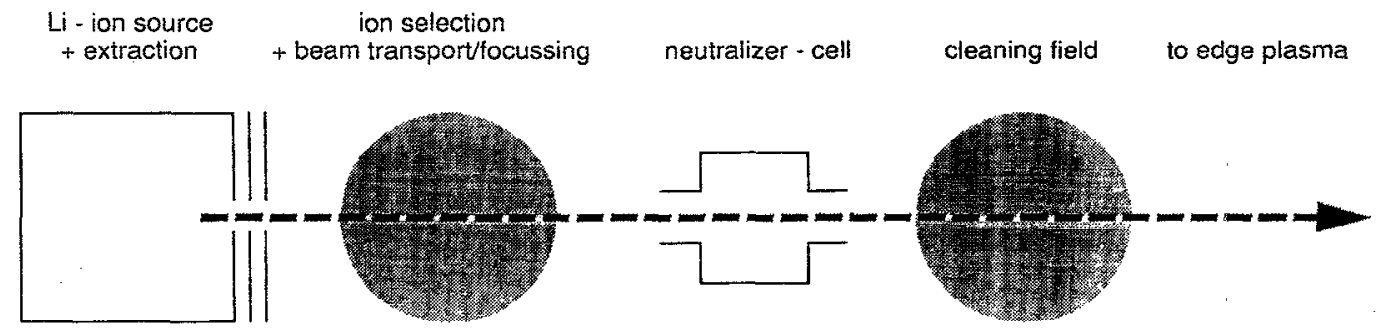

FIG. 1. Principal components of a fast neutral Li beam injector for application in Li-beam edge plasma spectroscopy.

ready utilized for detection of fully stripped light impurity ions in tokamak plasmas. ${ }^{12-15}$ However, as first shown in Ref. 16, impurity ion diagnostics in the edge plasma may better be carried out with fast neutral $\mathrm{Li}$ beams, since much more favorable signal-to-background conditions for the detection of fully and, in particular, incompletely stripped impurity ions can be achieved. Furthermore, the corresponding dominant line emission appears at comparably longer wavelengths, since electrons from $\mathrm{Li}$ are captured into higher excited states, which offers considerable experimental advantages.

The first successful application of Li charge exchange spectroscopy (Li-CXS) in a tokamak edge plasma concerned $\mathrm{C}^{5+}$ ion detection in TEXTOR, ${ }^{17}$ and important progress in this field has been made since. ${ }^{18} \mathrm{Li}$-CXS may as well be utilized for hydrogen ion detection in the edge plasma region. ${ }^{19}$ Since electron capture from fast $\mathrm{Li}$ atoms into multicharged ions is accompanied by negligibly small transfer of kinetic energy, profiles of such produced impurity emission lines can well serve for spatially resolved ion temperature measurements, as was recently demonstrated. ${ }^{20}$ In summary, fast $\mathrm{Li}$ beam spectroscopy provides a very versatile instrumentary for investigation of practically all relevant edge plasma properties, from which the physically relevant information can be derived without excessive plasma modeling. However, one has to keep in mind that the injected Li beam is rapidly attenuated and its excited-state composition is strongly changed along the atomic beam path through the plasma edge. ${ }^{18}$ This is not only of concern for absolute electron density determination via Li-IXS, but also for Li-CXS, since the relevant electron capture processes strongly depend on the ionization energy of the "active electron" initially bound to the injected $\mathrm{Li}$ atoms. Consequently, a full utilization of Li-beam plasma spectroscopy needs careful modeling of the $\mathrm{Li}$ beam attenuation and excited-state composition, ${ }^{4}$ as will be further explained in Sec. III.

\section{NEUTRAL LI BEAM PRODUCTION AND LINE EMISSION SPECTROSCOPY}

\section{A. Production of fast LI beams}

As sketched in Fig. 1, fast neutral Li beam production essentially involves the following elements:

(a) A high current $\mathrm{Li}^{+}$ion source with ion extraction and beam formation components, (b) a neutralizer cell for producing the fast $\mathrm{Li}$ atom beam,

(c) a deflector magnet for removal of remaining $\mathrm{Li}$ ions behind the neutralizer cell,

(d) neutral beam transport components toward the edge plasma to be diagnosed.

As will be shown below, the $\mathrm{Li}$ ion source is quite critical, while all other components pose no particular problems. For high-resolution plasma density measurements, neutral beam equivalent current densities of 0,1 $\mathrm{mA} / \mathrm{cm}^{2}$ might already be sufficient, but for Li-CXS at least $1 \mathrm{~mA} / \mathrm{cm}^{2}$ is necessary. Assuming an ion neutralization efficiency of typically $\geqslant 50 \%$ (cf. below), primary $\mathrm{Li}^{+}$ current densities have to be a factor of 2 higher than the above stated neutral current densities. For achieving a spatial resolution of typically better than $1 \mathrm{~cm}$, total $\mathrm{Li}^{+}$ion currents in the 1-10 $\mathrm{mA}$ range are needed, and a suffciently small emittance of the ion source and acceptance of the subsequent ion-optical systems have to be secured.

$\mathrm{Li}^{+}$ion beams from $\beta$-eucryptite emitters ${ }^{21}$ have been applied for Li-beam plasma spectroscopy by a number of groups. ${ }^{1-3,5,22}$ For $60 \mathrm{keV} \mathrm{Li}{ }^{+}$ion beam energy, currents of 3-5 mA from a 1 -in.-diam emitter surface have been reported. ${ }^{22}$ A detailed technical report is available on the ASDEX $\beta$-eucryptite type Li ion gun. ${ }^{23}$ While such ion sources are rather convenient because of their comparably long lifetime and high ion beam purity, they are severely limited in extractable total ion current with sufficiently small emittance. Consequently, the achievable ion current densities have so far not been higher than about 1 $\mathrm{mA} / \mathrm{cm}^{2}$. Moreover, there is no simple way for rapid chopping of the such produced ion beams, as would be necessary for Li-CXS applications.

Another way for $\mathrm{Li}^{+}$ion production utilizes plasma ion sources based on various types of low-pressure arc discharges fed by $\mathrm{Li}$ vapor together with auxiliary gases. ${ }^{24-26}$ Extracted ion current densities of $15 \mathrm{~mA} / \mathrm{cm}^{2}$ have been reported for a DUOPIGATRON source, however, without details on the related ion beam quality. ${ }^{27}$ At TEXTOR, $20-30 \mathrm{keV} \mathrm{Li}{ }^{+}$ion beams with typically $4 \mathrm{~cm}$ diameter and currents of well above $10 \mathrm{~mA}$ are produced from a reflex discharge ion source followed by a $55^{\circ}$ stigmatically focusing magnet. ${ }^{18}$ The mass-separated ${ }^{7} \mathrm{Li}$ ion beam is subsequently passed through a $\mathrm{Li}$ vapor-filled cell, and the resulting neutral $\mathrm{Li}$ atom beam travels another $2 \mathrm{~m}$ toward the TEXTOR plasma edge, with the remaining $\mathrm{Li}$ ions being magnetically deffected into a beam dump. There is 
still considerable potential for much better ion beam quality than so far achieved, if multiple-aperture extraction is applied instead of the more common single hole extraction geometry. However, all kinds of low-pressure arc sources suffer from limited cathode filament lifetime, Li vapor condensation and other technical problems, which concern both the ion source reliability and its operational lifetime (typically not more than 5-10 h). To minimize such difficulties, the very attractive features of ECR plasma ion sources such as their high ion currents, low emittance, and rather long lifetime ${ }^{28}$ can probably be utilized for $\mathrm{Li}^{+}$ion beam production, as well. A recently developed compact $2.45 \mathrm{GHz}$ ECR ion source ${ }^{29}$ is now adapted for $\mathrm{Li}^{+}$ion beam production, by applying different techniques of $\mathrm{Li}$ vapor feeding into the ECR plasma region.

Conversion of the fast $\mathrm{Li}^{+}$ions into neutral beams is routinely made via charge exchange in either $\mathrm{Li}^{1,18}$ or $\mathrm{Na}^{30}$ filled vapor cells. Given favorable conditions, with both alkali species neutralization efficiencies of well above $50 \%$ have been reported. $\mathrm{Na}$ is technically more convenient because of its sufficiently high-vapor pressure already at moderate neutralizer cell temperature $\left(\sim 220\right.$ vs $\sim 500^{\circ} \mathrm{C}$ for $\mathrm{Li})$, but special care is needed to avoid diffusion of the $\mathrm{Na}$ vapor from the exchange cell into the plasma region. On the other hand, if $\mathrm{Li}$ vapor is used for neutralization, the resulting $\mathrm{Li}$ diagnostic beam contains a thermal component made up from $\mathrm{Li}$ vapor effusing from the charge exchange cell. Excitation of this thermal $\mathrm{Li}$ atoms inside the plasma leads to LiI $670.8 \mathrm{~nm}$ background radiation which makes pulsed operation of the fast neutral $\mathrm{Li}$ beam mandatory.

\section{B. Spectroscopical techniques}

The essential quantities to be measured are the intensities and/or spectral shapes of line emission induced due to the $\mathrm{Li}$ atom beam interaction with the plasma constituents. To this purpose, light emitted from the interaction region has to be collected by a lens system and transported via fiber or lens optics to a spectroscopical detection system, which can involve visible/UV spectrometers (TEXTOR), interference filter-photomultiplier combinations (ASDEX, TEXTOR) and/or interference filterCCD camera combinations (TEXTOR). If necessary, the $\mathrm{Li}$ beam induced line emission can be separated from plasma background radiation by fast chopping of the diagnostic beam. Chopper frequencies of up to $500 \mathrm{~Hz}$ have been utilized with the setup at TEXTOR. Spatial profiling (i.e., measuring line emission intensity along the injected Li beam axis) can be achieved by an electrically driven mirror, to scan the line of observation along the beam direction (TEXTOR), by one- or two-dimensional observation systems like a CCD camera (TEXTOR), or by a number of photomultipliers monitoring the beam at different locations (ASDEX).

While the CCD setup provides excellent spatial resolution (typically $<1 \mathrm{~mm}$ ), the so far best time resolution ( $<1 \mu \mathrm{s}$ ) has been obtained with sets of photomulipliers. If it is necessary to subtract the background signal, the $\mathrm{Li}$ beam modulating frequency itself limits the achievable time resolution. The such obtained profiles have still to be corrected for effects due to the observation geometry. This is achieved by scanning along a $\mathrm{Li}$ diagnostic beam which is injected into the plasma vessel filled with low-pressure (typically $10^{-5}-10^{-4} \mathrm{mbar}$ ) $\mathrm{H}_{2}$ or He gas. Under the then given single collision conditions no appreciable attenuation of the beam will take place, which can be checked by a linear signal intensity dependence on the filling gas pressure. The such obtained intensity profiles include the radial dependences of all calibration-relevant quantities, e.g., solid observation angle, transmission of filters (taking also into account viewing angle-dependent Doppler shifts), vignetting due to apertures in the (scanning) observation system, and quantum efficiencies of the applied photon detectors.

\section{LI BEAM MODELING AND RELATED ATOMIC DATA BASE}

In order to obtain reliable quantitative diagnostic information from $\mathrm{Li}$ beam spectroscopy, the $\mathrm{Li}$ beam interaction with the plasma constituents has to be modeled. For collisions with fast $\mathrm{Li}$ atoms one has to regard not only the plasma electrons (as in the case of thermal $\mathrm{Li}$ beams), but also hydrogen and impurity ions, since the $\mathrm{Li}$ atoms can become excited as well as ionized in all of these collisional processes (impact excitation, ionization, and charge exchange). Consequently, these excited LiI states undergo both radiative and collisional deexcitation (the faster the beams, the more important becomes collisional deexcitation in plasma regions with higher density). The effective lifetimes of these excited states tend to smear out structures in the electron density.

As has been shown in Ref. 18, an appropriate model must assume that the occupation number for the $\mathrm{Li}(2 p)$ state [which is proportional to the measured $\operatorname{LiI}(2 s-2 p)$ photon intensity at $670.8 \mathrm{~nm}$ ), the plasma density $n_{e}$ and the occupation numbers of other $\operatorname{Li}(n l)$ states $N_{i}[i=1$ denotes $\mathrm{Li}(2 s), i=2 \mathrm{Li}(2 p)$, etc.] are interrelated by the following system of coupled differential equations:

$$
\begin{aligned}
& \frac{d N_{i}(z)}{d z}=\left\{n_{e}(z) a_{i j}[T(z)]+b_{i j}\right\} N_{j}(z), \\
& N_{i}(z=0)=\delta_{1 i} .
\end{aligned}
$$

Here a local plasma coordinate $z$ along the injected $\mathrm{Li}$ beam $(z=0$ at the entrance of the $\mathrm{Li}$ beam into the plasma) has been introduced as the independent variable. For the practical examples presented in Sec. IV, this coordinate $z$ along the neutral $\mathrm{Li}$ beam coincides with the small tokamak radius $r$. The density of each plasma component is expressed as the fraction of the electron density $n_{e}$ (for impurity-free plasmas $n_{p}=n_{e}$ ). Taking also impurity ions into account requires additional assumptions, e.g., an average $q(z)$ profile in combination with a model $Z_{\text {eff }}(z)$ profile. ${ }^{4}$ Coefficients $a_{i j}(i \neq j)$ in the rate equations refer to excitation and deexcitation processes for the $\mathrm{Li}$ atoms from state $j$ to state $i$, due to collisions with both the plasma electrons and hydrogen ions. 

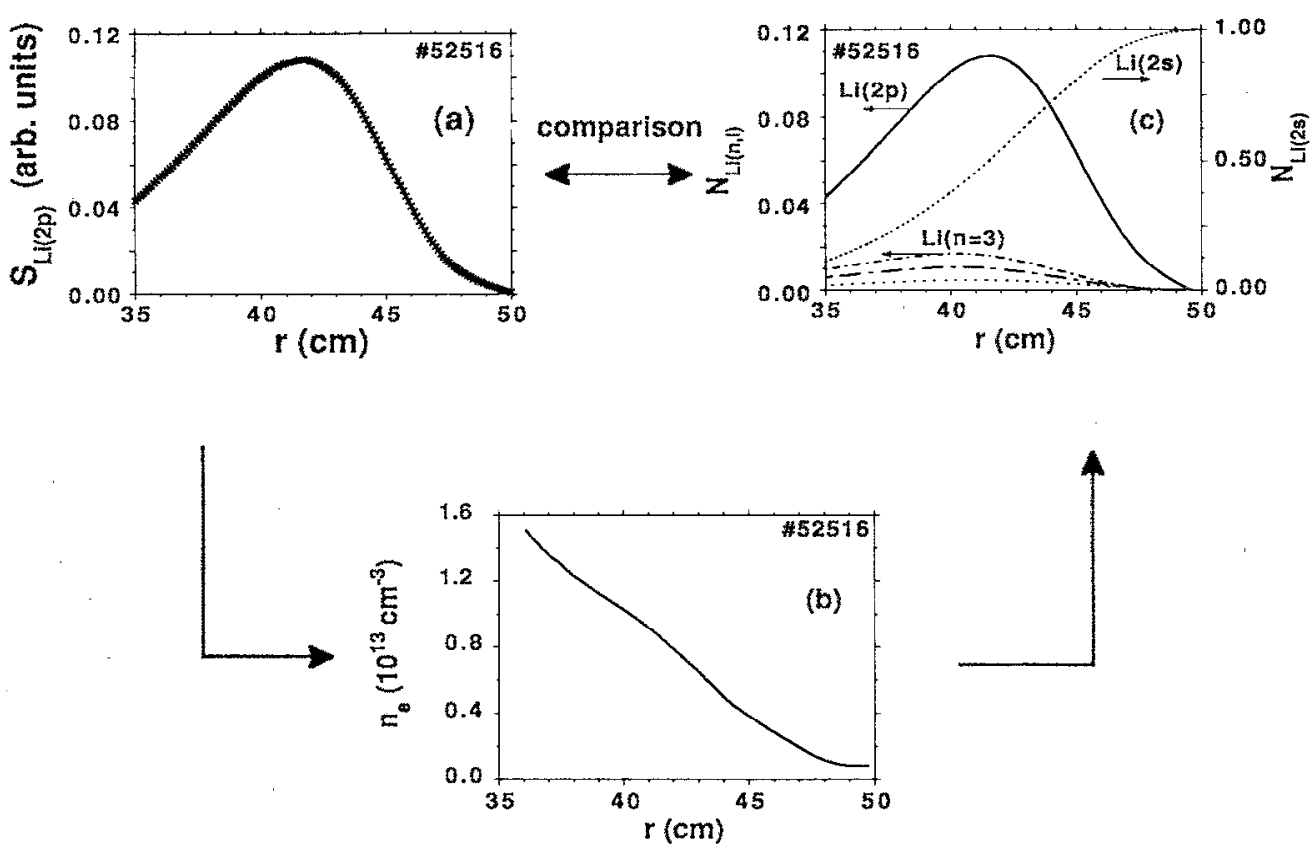

FIG. 2. (a) Typical radial LiI $670.8 \mathrm{~nm}$ emission profile (crosses) measured by $20 \mathrm{keV}$ Li beam spectroscopy during TEXTOR discharge No. 52516 . (b) Plasma electron density as reconstructed from the $\mathrm{Lil}$ emission profile in (a). (c) $\mathrm{Li}(2 s), \mathrm{Li}(2 p)$, and $\mathrm{Li}(3, l=0,1,2)$ components of the $20 \mathrm{keV} \mathrm{Li}$ beam, calculated via Eq. (1) for the electron density given in (b). The accuracy of the reconstruction method can be judged from a direct comparison of the $N_{2 p}$ population in (c) with the Lil $(2 s-2 p)$ emission intensity in (a), where $N_{2 p}$ has been added as a solid line.

Attenuation of particular Li states $i$ due to charge ex change and ionization in collisions with plasma particles and excitation as well as deexcitation to other bound states $j$ are included in coefficients $a_{i i}$. Finally, the spontaneous emission processes are described by coefficients $b_{i j}$. Since Eq. (1) takes also into account the finite lifetime of different $\mathrm{Li}$ levels as well as population of these levels via cascading, the calculated occupation number $N_{2}(z)$ of the $\mathrm{Li}(2 p)$ state is directly related to the measured $\operatorname{LiI}(2 s-2 p)$ emission profile, with the proportionality factor depending on the detection geometry. Of course, knowledge of absolute cross sections or related rate coefficients is required for all collisional processes between the different plasma constituents and all $\mathrm{Li}$ atomic states of relevance. Available experimental data on excitation, ionization, and charge exchange almost exclusively involve the $\mathrm{Li}(2 s)$ ground state only. Therefore, a critical evaluation of semiempirical and theoretical cross-section formulas for collisional processes also involving excited $\mathrm{Li}$ states has been carried out. The such produced "atomic data base for Li-beam plasma spectroscopy" has been compiled at the IAEA. ${ }^{31}$ It contains multiparameter fit formulas for each cross section. The rate coefficients calculated from these cross section data depend of course on the Li beam velocity, but (fortunately) only rather weakly on the plasma temperature $T(z),{ }^{19}$ because of which in most cases "standard $T(z)$ profiles" can be used for the evaluation.

If now a particular electron density $n_{e}(z)$ along the injected beam direction $z$ is introduced, the system of coupled differential equations (1) can be solved, resulting in the local population densities $N_{j}(z)$ of the different $\mathrm{Li}(j)$ states. However, the inverse task, i.e., reconstruction of absolute electron densities $n_{e}$ from only relatively measured Lil $670.8 \mathrm{~nm}$ diagnostic data is considerably more difficult. Emission profiles $\mathrm{Li}_{2 p}(z)$ as delivered from the $\mathrm{Li}$ beam diagnostic setup are directly proportional to the occupation number $\mathrm{N}_{2}(z)$ of the $\mathrm{Li}(2 p)$ state. A suitable reconstruction algorithm has recently been developed. ${ }^{4}$ It recovers the plasma (electron) density exclusively from scanned profiles of the relative LiI $670.8 \mathrm{~nm}$ emission intensity along the diagnostic beam direction. In principle, in this way any spatial variations in the electron density can be reconstructed, despite of the smearing-out effects mentioned above. ${ }^{32}$ More details on this novel algorithmus may be found in Ref. 4.

As an educative illustration, Fig. 2(a) shows a typical radial LiI $670.8 \mathrm{~nm}$ emission profile obtained with the 20 $\mathrm{keV} \mathrm{Li} \mathrm{injector} \mathrm{at} \mathrm{TEXTOR} \mathrm{(for} \mathrm{details} \mathrm{of} \mathrm{this} \mathrm{experimen-}$ tal setup cf. Ref. 18). The corresponding electron density as evaluated with the above described methods is given in Fig. 2(b). It shows a pronounced radial structure in the vicinity of the ALT II limiter position at $z=46 \mathrm{~cm}$. The accuracy of such determined density profiles is subject to errors in the applied atomic data base (estimated to about $\pm 20 \%$ in absolute $n_{e}$ magnitude, but only a few percent in the relative radial density profile), and direct experimental errors as signal/noise etc., for measuring the LiI emission line intensity along the injected $\mathrm{Li}$ beam (dominant error contribution to the relative radial $n_{e}$ dependence $\pm 10 \%$ ). Numerical errors of our reconstruction method amount to less than $3 \%$. In Fig. $2(\mathrm{c})$, the population of $\mathrm{Li}(n, l)$ excited states calculated from the density profile in Fig. 2(b) is shown. The high quality of our reconstruction method may be judged from a direct comparison of the $N_{2 p}$ popu- 


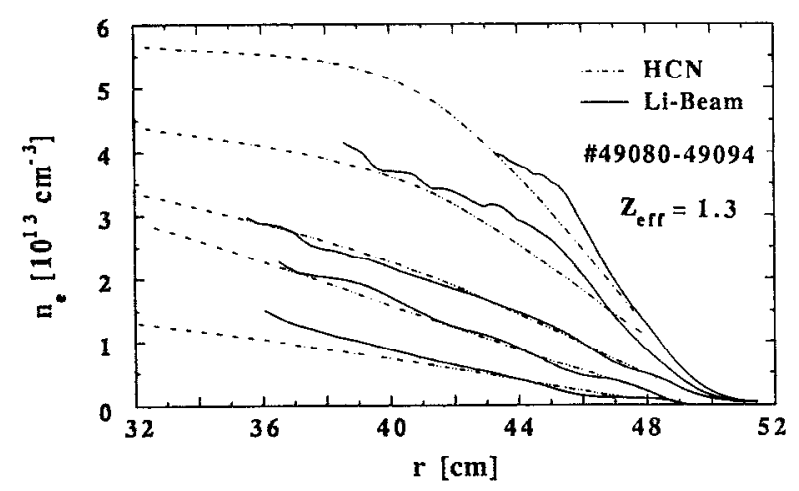

FIG. 3. Plasma electron densities as observed for a series of TEXTOR discharges. Comparison is mare between data obtained from Li-beam plasma spectroscopy (-) and HCN-laser interferometry (Ref. 33) $(-\cdots, \cdot \cdot)$.

lation in Fig. 2(c) with the $\operatorname{LiI}(2 s-2 p)$ emission intensity in Fig. 2(a).

\section{RECENT APPLICATIONS FOR TOKAMAK EDGE PLASMA SPECTROSCOPY}

\section{A. Electron density measurements}

Reconstruction of plasma edge density profiles from the spatial variation of $\mathrm{Li}(2 p-2 s)$ emission intensity has been carried out with the methods described in Sec. III. Corresponding measurements have already been carried out at TEXTOR and ASDEX. For example, edge density profiles have been compared for $\mathrm{OH}-, \mathrm{L}-$, and $\mathrm{H}$-discharge phases at ASDEX.,32 In the same context, the remarkable time resolution capability of $\mathrm{Li}$-IXS could be demonstrated by following the decay phase of an edge-localized mode (ELM). ${ }^{32}$

Regarding Li-IXS measurements at TEXTOR, in Fig. 3 we have compared a series of edge plasma electron densities ("density scan") as obtained by means of $20 \mathrm{keV}$ Li-beam plasma spectroscopy with independent density measurements by means of the TEXTOR HCN laser interferometer. ${ }^{33}$ Despite the large variation in plasma densities between different shots, the agreement between results of both kinds of measurements is rather good. Remaining discrepancies should be attributed to the different toroidal positions of both diagnostic systems, and especially to the fact that the spatial resolution of the $\mathrm{HCN}$ diagnostics is rather limited (the related data had to be obtained from Abel inversion of line-averaged electron densities derived at nine discrete radial positions, the two outermost ones being located at $r=40$ and $47.5 \mathrm{~cm}$, respectively).

\section{B. Impurity ion density measurements at TEXTOR}

Li-beam charge-exchange plasma spectroscopy ( $\mathrm{Li}$ CXS) makes use of electron capture processes according to Eq. (2) from neutral $\mathrm{Li}$ atoms into excited states of impurity ions, which then decay under emission of their char- acteristic lines. From the respective intensities the concentration of any particular impurity ions $\mathbf{A}^{q+}$ can be determined:

$$
\mathrm{A}^{q+}+\mathrm{Li} \rightarrow \mathrm{A}^{(q-1)+*}(n, l)+\mathrm{Li}^{+} .
$$

Since charge exchange with $\mathrm{Li}$ takes place with relatively large cross sections and ends up in comparably highly excited states of the impurity ions, which only to a small extent are populated by collisional processes with the plasma constituents, a favorable signal-to-noise ratio can be achieved. In the course of charge exchange with $\mathrm{Li}$ the kinetic energy transferred to the impurity ion is negligible. Therefore, the such induced impurity lines give a rather direct information on the spatial ion distribution in the edge plasma. If population of the different $\mathrm{Li}$ atomic states in the plasma along the complete beam path is known, the impurity ion density $n_{q}(z)$ can be calculated from the signal of the characteristic line radiation $S_{\lambda}(z)$ :

$$
S_{\lambda}(z)=k_{\lambda} v_{\mathrm{Li}} n_{q}(z) \sum n_{i} \sigma_{\lambda}^{i} .
$$

Here $z$ is the distance along the beam axis and $v_{\mathrm{Li}}$ the velocity of the $\mathrm{Li}$ atoms. The $n_{i}$ are the densities of the $\mathrm{Li}(n l)$ states to be derived from model calculations (see Sec. III) with known electron density and temperature and the measured absolute current density of the $\mathrm{Li}$ beam.

The $\sigma_{\lambda}^{i}$ are emission cross sections for the characteristic impurity lines produced as the result of electron capture from a $\operatorname{Li}\left(n^{*}, l^{*} \equiv i\right)$ atom into impurity ions $\mathrm{A}^{q+}$, ending up in atomic states $\mathrm{A}^{(q-1)+}(n, l)$. For electron capture from the $\operatorname{Li}(2 s)$ ground state these emission cross sections have been determined experimentally (cf. e.g., Ref. 34), whereas for capture from excited $\mathrm{Li}$ states the related cross sections have been calculated according to the "classical over the barrier model."35 The parameter $k_{\lambda}$ includes the wavelength-dependent sensitivity and the aperture of the optical detection system, and has to be determined experimentally. A detailed description of the experimental conditions at TEXTOR can be found elsewhere. ${ }^{18}$

The determination of both electron and impurity densities with a $20 \mathrm{keV} \mathrm{Li}$ beam has been employed at TEXTOR to contribute to the study of active edge cooling. During these experiments, the deliberate and well-adjusted puffing of neon gas into the plasma boundary was used to lower the electron temperature considerably in the outmost $10 \mathrm{~cm}$ of the plasma, while $T_{e}$ stays more or less constant in the hot center. ${ }^{36,37}$ As an example, we report on an ohmically heated discharge with a line-averaged central electron density of $2.4 \times 10^{13} \mathrm{~cm}^{-3}$ and central electron temperature of $1.0 \mathrm{keV}$, the plasma current being $350 \mathrm{kA}$ and the limiters placed at a minor radius of $46 \mathrm{~cm}$.

Neon has been injected during the time period of 0.8 $2.4 \mathrm{~s}$, where a steady state could be reached by adjusting the gas influx to a constant intensity of a $\mathrm{Ne}^{7+}$ spectral emission line. Figure 4(a) shows electron temperatures taken at $0.7 \mathrm{~s}$ (ohmic prephase, without neon) and at $1.5 \mathrm{~s}$ (edge cooling with neon injection) employing optical spectroscopy involving the interaction of a thermal helium beam with the plasma. ${ }^{38,39}$ It can clearly be seen that $T_{e}$ is 

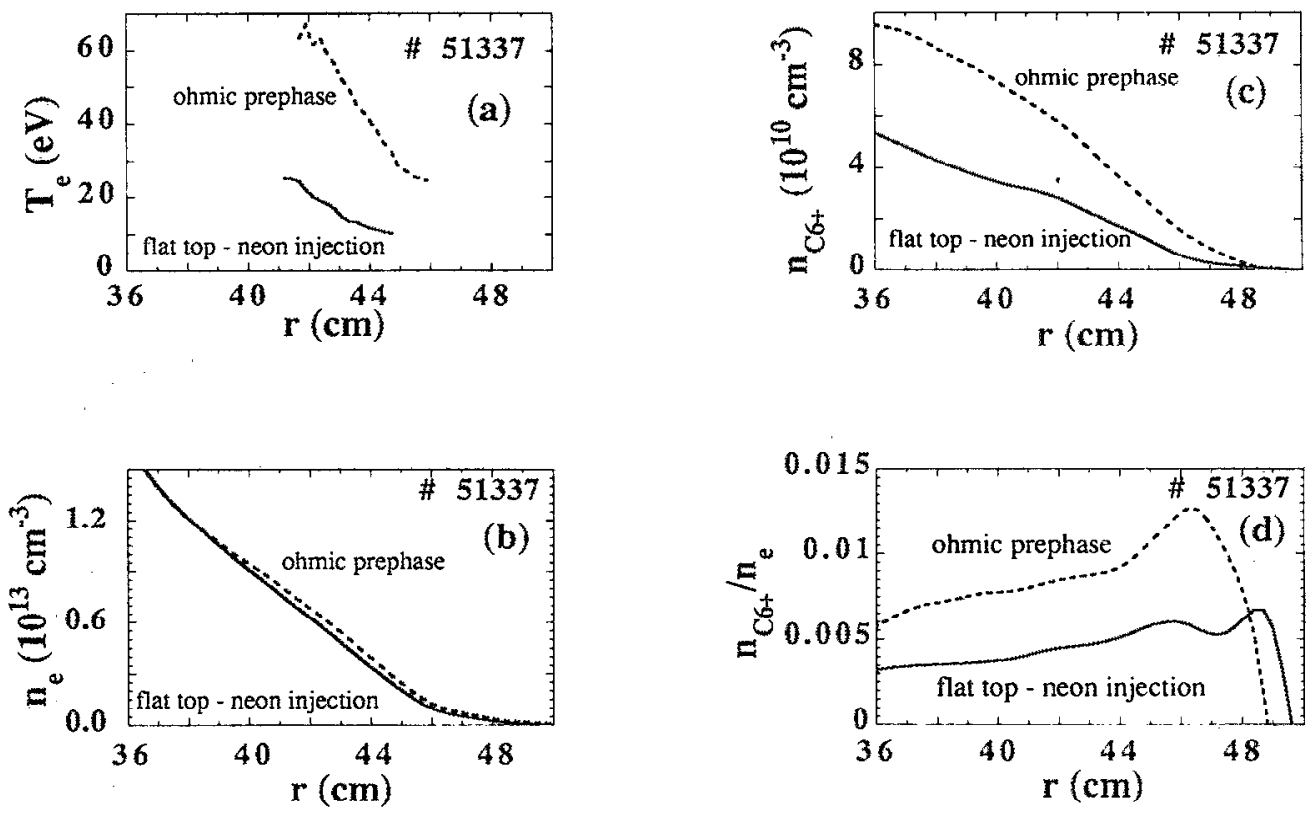

FIG. 4. (a) Radial electron temperature profiles during ohmic prephase $(0.375-0.875 \mathrm{~s})$ and fat top phase (1.125-2.125 $\mathrm{s})$, respectively, of TEXTOR discharge No. 51337. (b) Electron density profiles for TEXTOR discharge No. 51337 as defined under (a). (c) Radial $\mathrm{C}^{6+}$ profiles obtained by means of Li-beam plasma spectroscopy for TEXTOR discharge No. 51337 as defined under (a). (d) Relative concentration of $\mathrm{C}^{6+}$. Neon gas puffing during the time interval of $0.9-2.2 \mathrm{~s}$ causes lower $\mathrm{C}^{6+}$ concentration and a flatter slope in the investigated region.

reduced considerably by nearly a factor of 3 in the vicinity of the limiter radius, while its value at $r=0$ (not depicted, different method) even slightly rises from 1.0 to $1.2 \mathrm{keV}$. This somewhat contradictory behavior is due to an increased ohmic heating power induced by plasma detachment ${ }^{40}$ and, as a consequence, better particle confinement during the injection phase. In parallel, $Z_{\text {eff }}$ rises from 1.8 to 3.0 on the center axis. Neon cooling seems to be a method which selectively modifies the properties of the boundary layer while the influence on the overall performance of the center plasma is less pronounced. The physical mechanism responsible for this is electromagnetic line radiation especially of the edge-located lower ionization states of neon: For the shot under discussion, the total radiated electromagnetic power is $360 \mathrm{~kW}$ during gas injection (measured with a bolometer), while it is only 100 $\mathrm{kW}$ in the prephase. Compared to the total power of 400 $\mathrm{kW}$ launched into the discharge, nearly $90 \%$ of it is radiated isotropically into space and is thus prevented from being loaded onto limiter surfaces. Li-IXS shows that electron density profiles are not altered significantly by neon cooling [sce Fig. 4(b)], in contrast to density profiles of $\mathrm{C}^{6+}$ impurities measured by means of Li-CXS. Their absolute [Fig. $4(\mathrm{c})$ ] as well as relative concentrations [Fig. 4 (d)] are diminished by roughly a factor of 2 . The additionaliy measured reduction of the carbon release on the limiter surfaces by $35 \%$ may give an explanation: Lower electron temperatures lead to lower sheath potentials above the limiter surfaces and thus to reduced physical sputtering yields, being especially relevant to self-sputtering by highly ionized carbon states, ${ }^{20}$ where, e.g., a potential of $6 \times 3 k T_{e}$ would be given for $\mathrm{C}^{6+} \rightarrow \mathrm{C}$ sputtering.

\section{Impurity ion temperature measurements}

Application of $\mathrm{Li}-\mathrm{CXS}$ for temperature measurements of fully stripped $\mathrm{C}^{6+}$ ions in the boundary layer of the TEXTOR tokamak has recently been demonstrated and described in detail, ${ }^{20}$ by utilizing the same atomic collision processes [cf. Eq. (2)] as for impurity ion density determination. The impurity ion temperature can be deduced from spectral broadening of the resulting emission line radiation due to the velocity-dependent Doppler effect, assuming a Maxwellian velocity distribution of the emitting particles. However, other mechanisms leading to line broadening have to be taken into account, as outlined below.

From laboratory measurements ${ }^{34}$ it is known that after $\mathrm{C}^{6+}$-Li charge exchange the electrons are captured mainly into the $n=7$ and $n=8$ principal shells with high $l$ values, giving rise to optical radiation at $343 \mathrm{~nm}(7-6)$ and $529 \mathrm{~nm}$ (8-7), respectively. Because of more experimental convenience the latter transition has been used at TEXTOR and its spectral profile has been analyzed. For achieving a good spatial resolution across the boundary layer, at the moment still several reproducible discharges are necessary. By assuming a Maxwellian velocity distribution, the spectral line shape of a single transition $(n, l) \rightarrow\left(n-1, l^{\prime}\right)$ can be expressed by a Gaussian profile

$$
S(\lambda) \propto \exp \left[-\left(\frac{\lambda-\lambda_{0}}{\Delta \lambda}\right)^{2}\right]
$$

where $\lambda_{0}$ is the central wavelength of this profile and $\Delta \lambda$ its width. The ion temperature $T_{\text {imp }}$ can be obtained from a least-squares fit of the experimental data to relation (4): 


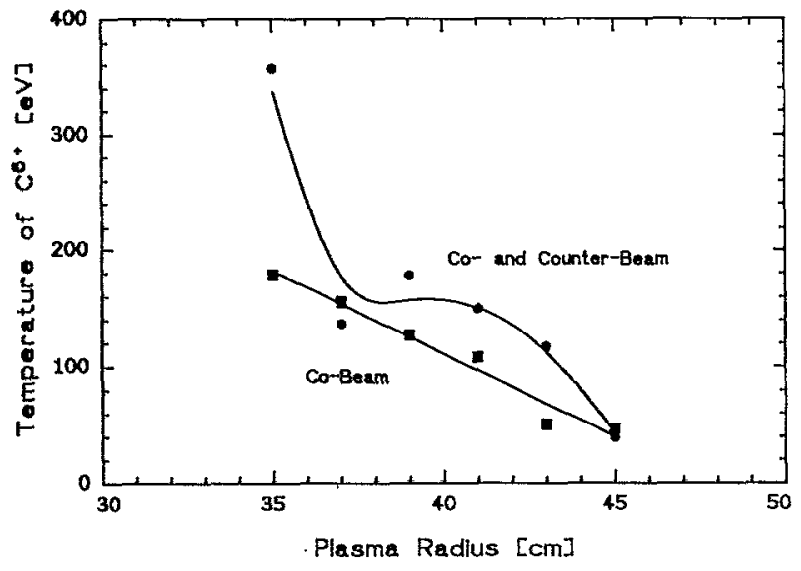

FIG. 5. $\mathrm{C}^{6+}$ temperature profiles as obtained for various conditions of a neutral beam-heated TEXTOR discharge with line-averaged central electron density of $3.5 \times 10^{19} \mathrm{~m}^{-3}$, which has been heated by two $1.8 \mathrm{MW}$ neutral deuterium beams in "co" and "counter" injection. The profiles have been measured during time intervals with a single and both injectors in operation, respectively.

$$
\Delta \lambda=\frac{\lambda_{0}}{c} \sqrt{\frac{2 k T_{\text {imp }}}{m}},
$$

with $m$ the mass of the impurity ion and $c$ the velocity of light. Besides the Doppler effect, two other processes have to be taken into account, namely, the Zeeman effect and collisional l-level mixing, which both cause additional broadening of the spectral profile. In tokamak edge plasmas magnetic fields of more than $2 T$ cause considerable Zeeman splitting of electronic energy levels, and the such further broadened spectral profiles would lead to systematically too high temperatures.

Therefore, so-called derating factors $\eta_{\text {Zeeman }}$ have been calculated dependent on the magnetic field strength and the measured temperatures. ${ }^{20}$ Alternatively, polarization filters could have been used in order to observe only those Zeeman components which experience the smallest Zeeman shift. Collisions of the $\mathrm{C}^{5+}$ particles resulting from charge exchange of injected $\mathrm{Li}$ atoms with $\mathrm{C}^{6+}$ with other plasma constituents can cause transfer between the different electronic $l$ states within the same principal $n$ shell. In tokamak edge plasmas this $l$-level mixing occurs on a faster time scale than the radiative lifetime of the respective state. Consequently, all allowed transitions between two $n$ shells will be observed simultaneously. The corresponding lines have to be superimposed to a sum profile by using the correct central wavelengths and relative intensities, from which a temperature-dependent corrective derating factor $\eta_{l_{\text {-mix }}}$ can be derived (for details cf. Ref. 20). The two nonthermal broadening mechanisms together with the apparatus profile of the applied spectrometer determine a lower limit of about $20-30 \mathrm{eV}$ for such temperature measurements. As an example, $\mathrm{C}^{6+}$ temperature profiles for beam-heated TEXTOR plasmas are drawn in Fig. 5. A discharge with line-averaged central electron density of $3.5 \times 10^{19} \mathrm{~m}^{-3}$ has been heated with two $1.8 \mathrm{MW}$ neutral deuterium beams directed in "co-" and "counter" injection, and impurity ion temperature profiles for time inter- vals with respectively one and both injectors in operation have been shown. Two main features are obvious. (1) The second ("counter") beam heats the plasma further up and (2) the gradient of $T_{\text {imp }}(r)$ gets steeper during counter injection, in parallel with the development of a more or less constant pedestal at $r \approx 40 \mathrm{~cm}$. These features may be interpreted as being due to improved plasma confinement in the hot center. ${ }^{41}$ In Ref. 20 it has been explained that the impurity ion temperatures as measured by Li-CXS in the here described way should more or less agree with the hydrogen ion temperatures at the same radial positions, whereas a considerable deviation from the corresponding electron temperatures may be given. This is caused by the relatively short equipartition time between impurity and hydrogen ions (typically several tens of $\mu \mathrm{s}$ ), as compared with the much longer equipartition times between impurities and plasma electrons (typically several $\mathrm{ms}$ ). The latter times are too long to permit achievement of equilibrium between electrons and impurities during the edge confinement times (typically $1 \mathrm{~ms}$ ) in TEXTOR.

It can be stated that Li-beam plasma spectroscopy has now reached a certain degree of maturity. However, to make it a more widely applicable (standard) diagnostic tool, further operational experience is desirable and technical efforts have to be devoted mainly to the construction of reliable, long-lived $\mathrm{Li}^{+}$ion sources for easy-to-operate neutral $\mathrm{Li}$ beam injectors.

\section{ACKNOWLEDGMENT}

The Austrian authors have been supported by Kommission zur Koordination der Kernfusionsforschung at the Austrian Academy of Sciences.

${ }^{1}$ K. Kadota, K. Tsuchida, Y. Kawasumi, and J. Fujita, Plasma Phys. 20, 1011 (1978).

${ }^{2}$ K. McCormick, M. Murmann, and M. El Shaer, J. Nucl. Mater. 121, 48 (1984).

${ }^{3} \mathrm{~K}$. McCormick and the ASDEX team, Rev. Sci. Instrum. 56, 1063 (1985).

${ }^{4}$ J. Schweinzer, E. Wolfrum, F. Aumayr, M. Pöckl, HP. Winter, R. P. Schorn, E. Hintz, and A. Unterreiter, Plasma Phys. Controlled Fusion 34, 1173 (1992).

${ }^{5}$ J. Fujita and K. McCormick, Proceedings of the Sixth European Conference on Controlled Fusion and Plasma Physics Moscow, 1973, p. 191.

${ }^{6}$ K. McCormick, M. Kick, and J. Olivain, Proceedings of the Eighth European Conference on Controlled Fusion Plasma Physics Prague, 1977, p. 140.

${ }^{7}$ E. Hintz and P. Bogen, J. Nucl. Mater. 128/129, 229 (1984).

${ }^{8}$ K. Kadota, H. Matsuoka, H. J. Ramos, S. Miyake, K. Tsuchida, and J. Fujita, J. Nucl. Mater. 128/129, 960 (1984).

${ }^{9}$ U. Rebhan, N. J. Wiegart, and H. J. Kunze, Phys. Lett. 85A, 228 (1981).

${ }^{10} \mathrm{~T}$. Oda and K. Takiyama, Proceedings of IAEA TCM on "Research using small tokamaks" Nagoya, Japan, 1987.

${ }^{11}$ F. Aumayr, M. Fehringer, and HP. Winter, J. Phys. B 17, 4185 (1984).

${ }^{12}$ V. V. Afrosimov, Y. S. Gordeev, and A. N. Zinov'ev, Sov. Tech. Phys. Lett. 3, 39 (1977).

${ }^{13}$ V. V. Afrosimov, Y. Gordeev, A. N. Zinov'ev, and A. A. Korotkov, JETP Lett. 28, 500 (1978).

${ }^{14}$ R. C. Isler, Phys. Rev. Lett. 38, 1359 (1977).

${ }^{15}$ R. J. Fonck, M. Finkenthal, R. J. Goldstone, D. L. Herndon, R. A. Hulse, R. Kaita, and D. D. Meyerhofer, Phys. Rev. Lett. 49, 737 (1982).

${ }^{16}$ HP. Winter, Comments At. Mol. Phys. 12, 165 (1982). 
${ }^{17}$ A. Pospieszczyk, F. Aumayr, H. L. Bay, E. Hintz, P. Leismann, Y. T. Lie, G. G. Ross, D. Rusbüldt, R. P. Schorn, B. Schweer, and HP. Winter, J. Nucl. Mater. 162-164, 574 (1989).

${ }^{18}$ R. P. Schorn, E. Hintz, D. Rusbüldt, F. Aumayr, M. Schneider, E. Unterreiter, and HP. Winter, Appl. Phys. B 52, 71 (1991).

${ }^{19} \mathrm{~F}$. Aumayr and HP. Winter, Ann. Phys. (Leipzig) 42, 228 (1985).

${ }^{20}$ R. P. Schorn, E. Wolfrum, F. Aumayr, E. Hintz, D. Rusbüldt, and HP. Winter, Nucl. Fusion 32, 351 (1992).

${ }^{21}$ J. P. Blewett and E. J. Jones, Phys. Rev. 50, 464 (1936),

${ }^{22}$ W. P. West, D. M. Thomas, E. S. Ensberg, J. S. deGrassie, and J. F. Baur, Rev. Sci. Instrum. 57, 1552 (1986).

${ }^{23}$ K. McCormick and M. Kick, Report IPP III/85, Max Planck Institut fir Plasmaphysik Garching, Germany, 1985.

${ }^{24}$ P. G. Weber, Rev. Sci. Instrum 54, 1506 (1983).

${ }^{25}$ H. Yamaoka, K. Kadota, K. Okuno, S. Masamune, S. Miyake, and J. Fujita, Proceedings of the Tenth Symposium on Ion Sources and IonAssisted Techniques Kyoto, Japan, 1986, p. 65.

${ }^{26}$ H. L. Bay, E. Dullni, and P. Leismann, Report Jül-2062 KFA Jülich, Germany, 1986.

${ }^{27}$ P. G. Weber and S. Masamune, Rev. Sci. Instrum. 57, 2032 (1986).

${ }^{28} \mathrm{Y}$. Jongen and C. M. Lyneis, in The Physies and Technology of Ion Sources, edited by I. G. Brown (Wiley, New York, 1989), Chap. 10.

${ }^{29} \mathrm{~S}$. Fiedler and HP. Winter, Rev. Sci. Instrum. 63, 2532 (1992).

${ }^{30} \mathrm{~K}$. McCormick, Report IPP III/82 Max Planck Institut fur Plasmaphysik Garching, Germany, 1983.
${ }^{31}$ F. Aumayr, R. K. Janer, M. Schneider, J. J. Smith, D. Wutte, J. Schweinzer, and HP. Winter, "Atomic data base for Lithium beam edge plasma spectroscopy," Report IAEA-INDC-NDS 267 IAEA Wien, Austria, 1993.

${ }^{32}$ F. Aumayr, R. P. Schorn, M. Pöckl, J. Schweinzer, E. Wolfrum, K. McCormick, E. Hintz, and HP. Winter, J. Nucl. Mater, 196-198, 928 (1992).

${ }^{33}$ H. Soltwisch, Phys. B 45, 225 (1989).

${ }^{34}$ E. Wolfrum, R. Hoekstra, F. J. de Heer, R. Morgenstern, and HP. Winter, J. Phys. B 25, 2597 (1992).

${ }^{35}$ A. Niehaus, J. Phys. B 14, 1161 (1986).

${ }^{36}$ U. Samm, J. Boedo, G. Bertschinger, K. H. Dippel, H. Euringer, K. H. Finken, D. Gray, D. Hillis, A. Pospieszcyk, D. Reiter, M. Tokar, and B. Unterberg, J. Nucl. Mater. 196-198, 633 (1992).

${ }^{37} \mathrm{U}$. Samm et al., Proceedings of the 14th International Conference on Plasma Physics and Controlled Nuclear Fusion Research, Würzburg, 1992.

${ }^{38}$ B. Schwcer, A. Pospieszczyk, G. Mank, and U. Samm, Proceedings of the 18th European Conference on Controlled Fusion and Plasma Physics, Berlin, 3-7 June 1991. and Europhys. Conf. Abstracts 15C Part IV, p. 361 .

${ }^{39}$ B. Schweer, G. Mank, A. Pospieszczyk, B. Brosda, and B. Pohlmeyer, J. Nucl. Mater. 196-198, 174 (1992).

${ }^{40}$ U. Samm et al, KFA-Report Jül-2123, March 1987.

${ }^{41} \mathrm{H}$. Conrads, H. Euringer, and D. Rusbüldt, J. Sci. Instrum. (in press). 